\title{
Barriers to Communication and Information Exchange in Patient Transfer and Its Consequences
}

\author{
Faramarz Pourasghar ${ }^{1}$, Nesa Kavakebi ${ }^{2}$, Jafar Sadegh Tabrizi ${ }^{3}$ \& Ahad Banagozar Mohammadi ${ }^{4}$ \\ ${ }^{1}$ Road Traffic Injury Research Center and Department of Health Services Management, Tabriz University of \\ Medical Sciences, Tabriz, Iran \\ ${ }^{2}$ Department of Health Services Management and Member of Student Research Committee, School of Health \\ Management and Medical Informatics, Tabriz University of Medical Sciences, Tabriz, Iran \\ ${ }^{3}$ Health Services Management Research Center, School of Health Management and Medical Informatics, Tabriz \\ University of Medical Sciences, Tabriz, Iran \\ ${ }^{4}$ Disasters and Emergency Medical Management Center, Tabriz University of Medical Sciences, Tabriz, Iran \\ Correspondence: Nesa Kavakebi, Department of Health Services Management, School of Health Management \\ and Medical Informatics, Tabriz University of Medical Sciences, Tabriz, Iran. Tel: 98-914-363-7319. E-mail: \\ kavakebi.n@gmail.com
}

Received: February 9, 2016 Accepted: March 29, 2016 Online Published: April 29, 2016

doi:10.5539/gjhs.v8n12p178 URL: http://dx.doi.org/10.5539/gjhs.v8n12p178

\begin{abstract}
Background \& Objectives: The patient transfer is multidisciplinary process and communication and information exchange are crucial for its successful accomplishment. The purpose of this study was to identify barriers that negatively influence communication and information exchange during the patient transfer between hospitals and the Center for Treatment Guidance and Information (CTGI) and to describe their consequences.

Methods: A qualitative study based on Focus Group Discussion (FGD) and semi-structured interviews were conducted. Three FGDs were carried out with 5 experts with years of experience working in the CTGI and 25 interviews with individuals involved in patient transfer process. Data were analyzed using content analysis method.

Findings: Three major themes including poor communication and information exchange at the CTGI, referring hospital, and receiving hospital were identified. The most important sub-themes at the level of CTGI were: the unavailability of accurate patient medical history and lack of confidence and different working process for patient admission in hospitals. At the level of referring hospital they were incomplete medical history, medical documents, vague patient transfer indications and lack of effective communication. At the level of receiving hospital they were lack of providing feedback, lack of mutual communication and incorrect report of available beds. Also four major consequences of poor communication and information exchange were identified which are managerial, clinical, economic and social consequences.
\end{abstract}

Conclusions: To overcome the barriers, there is a need for proper monitoring by accountable organizations, reviewing the protocols for patient transfer, an increase in inter-sector collaboration and improvement in communications infrastructure and collection of data.

Keywords: patient transfer, communication, information exchange, center for treatment guidance and information

\section{Introduction}

Transferring patients to continue treatment as well as facility distribution in specialty and subspecialty centers and facilitating the access to specialized services is inevitable (Coleman, 2003; Kripalani et al., 2007). Transferring patients is one of the most complex tasks due to the special condition of the patient, time constraints (urgency), a plurality of people involved in the transfer process in referring and receiving hospitals as well as the amount of logistics requirements (Haggstrom, Asplund, \& Kristiansen, 2009). To carry out these complex tasks, the minimum requirements and standards are required. Communication and clinical information exchange are the minimum requirements in optimal patient transfer (Behara et al., 2005; Goldhill et al., 2009). Information 
exchange between the various levels of service provided and also between various service providers is a vital factor in patient safety (Shanley, Sutherland, Stott, Tumeth, \& Whitmore, 2008).

However, the conducted studies have shown some shortcomings in communication, information exchange and quality in patient transfer (Gillman, Jacobs, \& Fatovich, 2014; Terrell et al., 2005). Data transfer and information exchange between healthcare providers in the health system is often inefficient and inadequate, and sometimes improper (Glintborg, Andersen, \& Dalhoff, 2007; Mesteig, Helbostad, Sletvold, Røsstad, \& Saltvedt, 2010; Payne, Kerr, Hawker, Hardey, \& Powell, 2002). In addition to inadequate and inaccurate information, there are communication barriers in the process of patient transfer. In a study by Shah and Payne, the most common communication barriers were unplanned and off-hours transfer, the absence of required efforts by hospital providers, time limit, lack of coordination and incoherent information (Payne et al., 2002; Shah, Burack, \& Boockvar, 2010).

Other barriers, which were discussed in a study by Mesteig and Coleman, are unwanted medication related events and medical events arising from the patient transfer, insufficient information exchange between health care centers and incorrect medication orders that had undesirable side effects for the patients(Coleman, Smith, Raha, \& Min, 2005; Mesteig, Helbostad, Sletvold, Rosstad, \& Saltvedt, 2010). Providing false medical history of the patient results in a lack of knowledge concerning the exact status of the patient, the emergency physicians in Carolina University Hospital were dissatisfied with the clinical information of over $40 \%$ of transferred patients due to incomplete reports and inadequate and non-standard information in transfer instructions(Platts-Mills et al., 2012). Dissatisfaction with the relationship between primary care centers and hospitals in the transfer of elderly patients was seen in a study by Dunnion (Dunnion \& Kelly, 2008).

Although patient transfer has some advantages, it can have some disadvantages such as mortality (Odetola, Bruski, Zayas-Caban, \& Lavieri, 2016). Incomplete clinical information and lack of coordination could adversely affect the quality and the continuity of patient care, which could result in potential injuries to patients (Coleman, 2003). The aforementioned injuries can lead to an increased use of services and rising costs (Cwinn et al., 2009; Griffiths, Morphet, Innes, Crawford, \& Williams, 2014).

Physicians and supervisors who authorize patient transfer, are the main medical staff in referring and receiving hospitals that play an important role in communication and information exchange during patient transfer process. The referring Physician is responsible for tasks such as completing transfer forms and keeping in touch with the receiving Physician. The receiving Physician is responsible for negotiating and consulting with the referring Physician and confirming the patient transfer form after admitting the patient in the receiving hospital. Supervisors are responsible for coordination between referring and receiving hospitals. In Iran, the process of coordination between referring and receiving hospitals is the responsibility of the Center for Treatment Guidance and Information (CTGI). CTGI is one of the units affiliated with the deputy of treatment affairs of the University of Medical Sciences, which there is a CTGI in each province. CTGI was founded to facilitate the transfer process, to monitor and follow-up the patient pathway and to organize any kind of transfer between all public and private hospitals. Ministry of Health and Medical Education (MOHME) protocols describe the functional structure and position of the CTGI, so the CTGI is responsible for obtaining admission on behalf of all patients who need to be transferred to public or private hospitals. In addition, inquiring for bed availability in university and non-university hospitals is one the tasks of CTGI (Ministry of Health \& Medical Education, 2007)

Only a few studies have been done on the patient transfer process in Iran. The literature review showed no specific qualitative study on the barriers regarding communication and information exchange in patient transfer from the perspective of process owners. Since the process of the patient transfer is multidisciplinary task and is done by different people in referring and receiving hospitals and CTGI. Therefore clarifying the barriers of communication and information exchange in patient transfer from the perspective of process owners seems to be necessary. Hence, the present study was conducted to examine the patient transfer barriers and barriers from above-mentioned perspective using the qualitative research approach in Tabriz University of Medical Sciences.

\section{Methods}

This was a descriptive study design with a qualitative approach carried out in Eaest Azerbayjan province of Iran in 2014. Settings of study were CTGI, one referring hospital and six receiving hospitals. CTGI is responsible for the admission and bed availability for the patients who need to be transferred in every university of medical sciences in Iran. CTGI is made up of Physicians and clinical care experts who are in the center round the clock. Hospitals supervisors and Physicians coordinate the transfer of qualified patients through phone. In this study, CTGI is the coordinator of patient transfer, Razi hospital is the referring hospital, picked from among the university affiliated hospitals of the towns in east Azerbaijan as it had the greatest number of yearly patient 
referrals, and six teaching hospitals in Tabriz with the greatest number of yearly admissions are the receiving hospitals. The receiving hospitals included three general hospitals and three specialized hospitals (Table 1).

Table 1. Characteristics of the study settings

\begin{tabular}{|c|c|c|c|c|c|c|c|c|}
\hline $\begin{array}{l}\text { Hospitals } \\
\text { and } \\
\text { centers }\end{array}$ & CTGI & Imam Reza & Razi & Shohada & Sina & $\begin{array}{l}\text { Shahid } \\
\text { Madani }\end{array}$ & Taleghani & Alzahra \\
\hline Location & Tabriz & Tabriz & Tabriz & Tabriz & Tabriz & Tabriz & Tabriz & Tabriz \\
\hline Type & Governmental & Governmental & Governmental & Governmental & Governmental & Governmental & Governmental & Governmental \\
\hline Specialty & ------- & General & General & Orthopedics & General & Cardiovascular & General & $\begin{array}{l}\text { Obsterics and } \\
\text { Gynocology }\end{array}$ \\
\hline $\begin{array}{l}\text { Active } \\
\text { beds }\end{array}$ & ------ & 600 & 130 & 250 & 260 & 255 & 118 & 160 \\
\hline
\end{tabular}

Thirty people participated in this study, selected through an intentionally sampling method for individual interviews and Focus Group Discussions(FGD), including five emergency head nurses, ten hospital supervisors, six specialists (an emergency medicine specialist, a gynecologist, a neonatal specialist, a forensic and poisoning specialist and a surgeon), three general practitioners, five senior residents (two internal residents, a cardiology resident, a surgical resident and a neurosurgery resident) and a CTGI expert. We tried to select participants with the richest information in order to provide the researchers with appropriate information in the selection process.

We held meetings with CTGI staff and the head of the emergency ward of the main receiving hospital in Tabriz to select participants with the richest information about the patient transfer barriers between the hospitals based on the selection criteria. Inclusion criteria included selecting those involved in the coordination of transferring process between the hospitals, at least one year of experience working in their respective workplace and willingness to participate in interviews.

Qualitative methods including Focus Group Discussions (FGD) and semi-structured qualitative interviews were used for data collection. The organizers of FGD meetings and interviews were a moderator, a note taker and an observer. The moderator in addition to explaining the objectives, led the discussions, and the note taker recorded the discussions and the observer controlled the meeting and time. Meeting content was based on an interview guideline designed using the literature review on communication and information exchange in the process of patient transfer and also the opinions of experts. The topic guide's validity was confirmed by two experts in qualitative research. Interview questions and focus group discussions focused on participants' experiences in the coordination of patient transfer, barriers to effective communication, barriers on exchange and sharing patient information, patient transfer and admission challenges, difficulties of bed availability and the implications and consequences of poor communication and information exchange. Interviews were conducted in pre-arranged coordination and after obtaining written informed consent. Each interview and discussion lasted 30-90 minutes. Interviews continued until saturation of the information that is up to the stage that the researchers felt no new information could be obtained. In total, three FGD meetings were held and 23 interviews were done. The participant interviews were recorded if they agreed. Within the 24 hours after the interviews, the recordings were transcribed word by word. It should be noted that if the participants did not agree to have the interview recorded, the researchers only took notes.

Content analysis was used manually for data analysis after transcribing recordings word by word. The interviews texts were analyzed in several steps. First, the texts were reviewed several times to obtain a general understanding of the interviews. Then texts related to the experience of participants, in accordance with the objectives of the study, were divided into meaningful units, sentences or phrases. Meaningful units were then summarized and coded. All codes representing the same concept were put in the same category. Various codes were compared in terms of their differences and similarities. Coding processes involved the identification and extraction of the initial codes, identifying themes (inserting the initial code extracted into the related themes), reviewing and completing the identified themes, naming and defining themes, achieving agreement between the two coders. The disagreements were resolved through discussion and in case of disputes, item removed. Inter coder reliability was measured by Cohen's un-weighted kappa. For subthemes of "The barriers of communication and information exchange in patient transfer" kappa was $=0.73$ (Confidence Interval $=0.95$, Standard Error=0.14), demonstrating good level of inter rater agreement between coders. For subthemes of "The 
implications of poor communication and information exchange in patient transfer" the kappa was calculated $=0.71$ and showed good level of inter rater agreement too (Confidence Interval $=0.95$, Standard Error $=0.18$ ).

Respondent validity was used for data rigor. Participants' remarks were summed up and repeated to the participants at the end of the meeting. The participants were asked whether the summarized text reflects the discussion and the accuracy of the researchers' notes and perceptions were confirmed by the participants.

Ethical considerations included obtaining approval from the ethics committee of Tabriz University of Medical Sciences, introducing the study objectives to the participants both in written and oral forms, obtaining informed consent, reminding the right to withdraw from the study at any time, retaining the participants' right to demand the results, keeping the information confidential and presenting the results without the names of the participants.

\section{Results}

The findings showed that there were barriers that negatively influence the communication and exchange of information on each of the three levels of CTGI, referring hospital, and receiving hospital (Table 2). In this qualitative study, three major themes (poor communication and information exchange at each of the levels), and seventeen subthemes on the barriers of communication and information exchange were identified. Poor communication and information exchange means any deficiencies and shortcoming in communication and information exchange among three mentioned level.

The consequences of communication and poor information exchange were classified into four major themes, including the managerial, clinical, economic and social implications, and eleven subthemes (Table 3).

Table 2. The barriers of communication and information exchange in patient transfer

\begin{tabular}{ll}
\hline Themes & Subthemes \\
\hline & The unavailability of accurate patient medical history \\
& Providing incorrect medical history \\
Poor communication and information exchange & Physicians' lack of confidence in provided medical history \\
at the CTGI & Different working process for patient admissions \\
& The patient transfer by CTGI order without coordination \\
& with the receiving hospital \\
& Not providing or providing incomplete medical history \\
Poor communication and information exchange & Vague declaration of patient transfer indications \\
at the referring hospital & Incomplete delivery or non-delivery of medical documents \\
& Lack of effective communication \\
& Disregarding the rules of patient transfer \\
& Not providing feedback to CTGI \\
& Lack of mutual communication among hospitals \\
& No phone consultation Physicians for information \\
exchange & The barrier of access to resident Physicians \\
Poor communication and information exchange & Lack of coordination among resident Physicians \\
at the receiving hospital & The lack of appointed individuals responsible for \\
admission & Incorrect report of available beds \\
\hline
\end{tabular}

Table 3. The implications of poor communication and information exchange in patient transfer

\begin{tabular}{ll}
\hline Themes & Subthemes \\
\hline \multirow{3}{*}{ Managerial Implications } & Dissatisfaction with the performance of different transfer levels \\
& Not transferring the patient to the qualified hospital \\
Clinical Implications & Interruption in coordination of admission \\
\hline
\end{tabular}




\section{Delay in treatment}

Not stabilizing the patient

Secondary damage and death

Economic implications

Duplicating tests and radiographies

The cost of patient transfer by ambulance

Undermining the mutual trust of transfer levels

Social implications

Uncertainty of the patient and their relatives

\subsection{Barriers with Communicating and Information Exchange in Patient Transfer}

\subsubsection{Poor Communication and Information Exchange in CTGI}

One of the barriers in communication and information exchange was the unavailability of accurate patient medical history which must be provided to the receiving hospital by CTGI and the providing of false medical history to the receiving hospital to increase the chance of the patient's admission. In this regard, one participant said, "sometimes, we ourselves make diagnosis due to the inaccuracy of medical history in the CTGI or we have to give false history to the hospital but we do not change them because it has responsibility for us, but we change the history in a way or use the literature to be accepted in the receiving hospital because staying patient in extra bed in equipped hospital like Imam Reza Hospital is better than staying at a hospital without sufficient equipment in a town..."

Some participants believed, "the Physicians in receiving hospitals do not rely on the medical history provided by the CTGI to the receiving hospital, and sometimes, the difference between history provided to CTGI and the history reflected by the receiving hospital is so great that the Physicians are not reliant on information provided from the CTGI, although the CTGI just transmitted the history provided by referring hospital. Therefore, the criterion for patient admission by the Physicians in the receiving hospital is the medical history provided directly by the Physician involved in referring the patient. The following is what a participant said in the CTGI: "It is a true that the Physicians in both referring and receiving hospitals talk on the patient's medical history because some Physicians in receiving hospital decide to accept or reject when they talk with the Physician in referring hospital. In other words, they do not believe in us or the accuracy of our words ..."

Another barrier in CTGI is different working processes in hospitals, especially in non-teaching hospital at the time of request for acceptance by the center. Different working processes can lead to a time-consuming admission process.

One participant explained it this way: "We got in touch with several people for admission of a patient with neurological barriers. At first, we talked with the Physician in receiving hospital and we got scientific confirmation. Then he asked us to call nursing room to confirm bed availability, they told us to call the emergency department resident physician's room to do the necessary coordination and finally, emergency department physician said that there was no bed available in Intensive Care Unit (ICU) and suggested us to consult with nursing room to transfer the patient at our own risk, if the level of Glasgow Coma Scale (GCS)) is normal."

The staffs believe that patient transfer in which CTGI is informed but there is no coordination with the receiving hospital from the CTGI occurs when the CTGI anticipates that the receiving hospital will not admit the patient, but the patient cannot be transferred to other hospitals but the staff of receiving hospitals considered the CTGI reasoning unjustified. In this regard, one participant explained: "CTGI is a center to coordinate the patient transfer to other hospitals but it is turned to Center for Treatment Guidance just to Imam Reza Hospital".

\subsubsection{Poor Communication and Information Exchange in Referring Hospital}

According to the interviewees, the medical histories provided for patients needing to be transferred were sometimes incomplete, unscientific and, in some cases, vague and fabricated. In addition, Physicians sometimes deliberately do not provide the correct information but sometimes they are not capable of making the correct diagnosis. One of them said, "due to non- transparent patient transfer indication by the referring hospital, the patient who has been transferred to orthopedic hospital with orthopedic barrier is again transferred to another hospital for ruling out surgery and neurosurgery conditions, but it is process which must be done first."

Due to a lack of awareness about patient medical treatment because of lack of patient medical records and consultation sheets, the communication and information exchange stops between referring and receiving hospitals and causes barriers in the treatment process of the patient. One participant said, "There was a case in 
which a patient with stroke was transferred without medical history in his medical records. We asked the patient if he had taken aspirin or not, he would not remember, neither would his relatives..."

If the patient transfer was canceled for any reason by the referring hospital, the receiving hospital would not be informed and the designated bed would be suspended. There are also some non-educational hospitals, private and social security hospitals in particular, that transfer the patient to a hospital without transfer forms because they do not want the hospital's statistics to show more patient transfers.

\subsubsection{Poor Communication and Information Exchange in Receiving Hospital}

There was poor communication between some receiving hospitals and the CTGI. For example, the referring hospital developed a mission form to ensure that patient admission was done successfully, but no information regarding the acceptance or rejection of the patient was given by the receiving hospital and the communication chain was completely cut off. The communication between the referring and receiving hospitals was also cut off after patient transfer. A participant said, "Fortunately or unfortunately, we get no feedback on patient transfer or admission in the hospital. For instance, if we know $70 \%$ of the transferred patients have no indications or they are not stabilized before transfer, we can do interventions needed easily and evidence-based."

In addition, due to the high workload of Physicians, sometimes there was no phone contact between Physicians in referring and receiving hospitals and those who were responsible for patient admission were not specified. In the absence of Physicians, senior residents were responsible for the patient's admission. Difficult access to residents was one of the major barriers. A participant described: "In some cases, we cannot find residents, for instance, it sometimes took us 30-40 minutes to find the resident because they were either somewhere in Imam Reza hospital where no mobile signal coverage or they are some residents for instance internal medicine residents who cover emergency room and general surgery wards simultaneously. Sometimes, finding residents sounds like a great tragedy for us..." There was inadequate interaction among some residents in receiving hospitals, as one participant said, "we got patient admission from resident 1 for transferring patient, then the resident shift finished before the patient transferred and the resident left the hospital, resident 2 in the receiving hospital did not admit the patient saying that no coordination was done with him..."

Not appointing a dedicated person with clear responsibilities for admission was another barrier that led to poor communication and information exchange. A participant explained the barrier as such: "Each time a different person from different ward contacted with CTGI, there is no identical procedure for people to contact the CTGI. These people do not have any information on patient condition and even they themselves have not seen the patient in most cases. As a result, we emphasized on getting the information because they do not know what to say ..."

Some hospitals do not report the number of available beds to CTGI. Although there were some beds available in some hospitals, their staff did not report the exact number of them. One participant at a receiving hospital explained the reason for doing so: "The patient who is transferred is coming from a hospital, despite that the referring hospital has minimum facilities, but patient with accident are brought from street and has priority to previous one. We sometimes predict these types of patients and try to have some beds available for them and it is the reason for not giving the exact number ..."

\subsection{Implications of Poor Communication and Information Exchange}

Implications of poor communication and information exchange were categorized into the four main classes including of managerial, clinical, economic and social implications.

\subsubsection{Managerial Implications}

Coordinator levels of patient transfer were not satisfied with the performance of CTGI. One participant explained, "As hospitals say that there is no bed available, CTGI is convinced, and they themselves may not want to report the beds available. The duty of CTGI is to monitor their accuracy, since it is named guidance center, it should guide ..."

Not transferring the patient to a qualified hospital and finally retransferring patients to other hospitals due to the lack of transfer indication is another implication of management. The duration of patient admission process increased because of ineffective communications, and the coordination for admission of emergency patients was interrupted. A participant said, "When the transferring Physician does not accept to contact with the receiving Physician, and a person other than Physician gives the patient medical history, a lot of time spends to coordinate the admission." 


\subsubsection{Clinical Implications}

Poor communication and information exchange leads to discontinuity and delay in patient treatment. The stabilization of the patient is not done based on the Physician's order. In this regard, one participant said, "a correct stabilizing of patient is not based on the receiving Physician expects. Some patients transferred intubated but without Ambubag, intubation tube in stomach or incorrect and subcutaneous inserting of IV line." Secondary injuries and death of patients transferred are some other implications; an interviewee stated a concrete example of this: "The referring hospital put subcutaneous IV line and patients was transferred along with a patient carrier, the patient had a seizure in the ambulance and the patient carrier injected diazepam subcutaneously to the IV line. Since IV line had been put incorrectly under skin, diazepam piled up and eventually patients died due to successive seizures."

\subsubsection{Economic Implications}

Sometimes, the laboratory tests and radiological exams done in referring hospitals were not sufficient enough to be accepted by the admitting Physician. The laboratory tests were repeated in the receiving hospital, and this imposed excess costs on the patient. The expense for patient transport by ambulance had to be paid by the patient. According to one participant, "the cost of patient transport by ambulance was three times more than as it used be, the family members who are not able to afford, they themselves have to transfer the patient, in which the patient's life is at risk because of lack of money."

\subsubsection{Social Implications}

Some participants believed that there was no relationship based on mutual trust between CTGI and the hospitals. No transparency in communication channels and a lack of coordination in patient transfer led to the patient's and his relatives' uncertainty.

\section{Discussion}

\subsection{Information Exchange}

Not providing or providing incomplete and vague medical history, incomplete delivery of medical records and pending test results were considered as obstacles and difficulties in patient transfer from the stakeholders' viewpoints in this study. The findings of this study are consistent with some studies that expressed barriers such as transferring patients without relevant documents, uselessness and non-applicability of the documents, and also not completing information or incomplete processing of information (King et al., 2013; Terrell \& Miller, 2006). Providing the patient's accurate and complete medical record is an effective strategy to ensure the quality of patient transfer (Jeffs, Lyons, Merkley, \& Bell, 2013). It seems that lack of knowledge on the importance of information, lack of awareness on the main components of the patient's medical history and various expectations of the specialists can be the reasons for providing incomplete history and non-delivery of related documents. The conducted studies have also shown that different attitudes of home care and hospital nurses toward the required information and expectations of professionals led to not providing the expected information in patient transfer (Apker, Mallak, \& Gibson, 2007; Olsen, Ostnor, Enmarker, \& Hellzen, 2013).

One of the reasons for providing incomplete medical history and noncompliance of medical history and vital signs at patient arrival to hospital is to increase the chances of success in admission. They want to convince the hospital to admit the patient by showing the critical condition of patient. Of course, the reverse may also occur, in which hospitals may report that the patient's general condition is good so that the receiving hospital does not avoid the admission. King et al. also showed contradictory information provided with the patient's clinical status arriving hospital (King et al., 2013) This paradox has created distrust among hospitals and leaves hospitals without the required preparations completed for patient admission, such as obtaining the medical equipment needed. This will negatively affect patient and would lead to delay in treatment.

The evidence emphasizes that medical information must be transferred accurately, systematically and coherently, so that this information can be considered as a directing map for the receiving center (Thraen, Bair, Mullin, \& Weir, 2012). Obviously, knowledge on the standard methods of providing medical history and having a common vision can help to solve the barrier of contradictory information. Standardization of the elements of information can also be effective regarding the quality and safety of patient transfer (Halasyamani et al., 2006).

Providing incorrect medical history is also observed in the performance of CTGI, as well as the hospitals. The main task of CTGI is to facilitate admission for the patients through coordination and communication between hospitals and obtaining relevant information. However, sometimes referring hospitals do not provide the CTGI with sufficient and credible information. Regarding the comments of participants, there, on the other hand, may not be a relationship based on mutual trust fully between CTGI and hospitals. Therefore, CTGI sometimes 
presents the medical history in a way to obtain admission. The CTGI staff believes that this is done for the benefit of patients. However, providing correct information and mutual trust is essential. It is the trust-based relationship that improves collaboration among people in an organization. Lack of confidence in the accuracy of the information provided by the nurses at the receiving center was also expressed as one of the barriers in the study conducted by Olsen et al. (2013).

CTGI attempts to do some tasks, such as repeated calls to referring hospital for obtaining additional information due to lack of sufficient information on the clinical condition. These activities are time-consuming and may lead to delays in patient treatment. There is no easy access to the people responsible for the patient transfer in order to obtain information through repeated calls and they are not often interested in going back to patient's information (King et al., 2013).

The use of new technologies can offer opportunities for the exchange of accurate and timely information. Experiences of other countries have shown that electronic data transfer leads to reduction in the loss of information and increases transparency in the responsibility of each employee (Patterson, Roth, Woods, Chow, \& Gomes, 2004). Web-based systems lead to accurate and timely exchange of information and reduce the time used for gathering information. Other features of a successful patient transfer system include increasing coordination between medical centers and eliminating unnecessary calls and patient transfers, resulting in an increase of staff satisfaction and reduce of staff workload (Hustey \& Palmer, 2010, 2012).

\subsection{Communication}

Not appointing particular individuals for admission and calling various people in CTGI for admission led to providing incorrect medical history and definitive diagnosis, and also the incorrect information is transferred to the receiving hospital as well. The receiving hospital believes that incorrect information is originated in CTGI because of not seeing the patient and the lack of knowledge on the general condition of the patient can cause inability to answer questions related to the patient (Olsen et al., 2013). In CTGI, some questions are asked for definitive diagnosis in patient transferred by transferring hospital. This, in some cases, is done using pressure on physicians either directly or indirectly. In such cases, the Physician may suggest a diagnosis that is not the final one and leads to bias and inaccurate performance. Communication and direct contact of physicians with each other is often the best way to organize and share information and make decisions about the patient treatment process (Warren, Fromm, Orr, Rotello, \& Horst, 2004) Therefore, any information exchange on the details of the patient clinical condition should be made by hospital Physicians. To do so, it is necessary to have mechanisms based on the responsibilities and clear expectations from physicians and staff. One of these mechanisms can be legal requirements to appoint particular individuals to coordinate and monitor the patient transfer (Patterson et al., 2004). As the patient transfer protocols consider the hospital supervisor responsible for coordinating the patient transfer with CTGI, they also consider the referring and receiving Physicians responsible for the exchange of clinical information and patient medical history (Ministry of Health \& Medical Education, 2007). Poor communication and individual responsibility are the barriers in the patient transfer process in the studies conducted by other researchers (Abraham \& Reddy, 2010; Horwitz et al., 2009). A qualitative study conducted to describe the mechanism of patient transfer at the University of Michigan reported the difficulties in areas of determining the patients eligible to transfer, determining the destination hospital, negotiating and communicating on patient transfer (Bosk, Veinot, \& Iwashyna, 2011).

Access to residents is through mobile phone in big hospitals. However, no mobile signal in some wards on the one hand and coverage of several wards by few residents on the other hand, disrupt the quick access to residents and lead to delays in responding to a request for patient admission. Some other communication methods, such as using pagers, may help access residents at any position and in any part of the hospital.

Based on the patient transfer instructions, hospitals are required to notify CTGI in writing the number of special beds availability three times a day (Ministry of Health \& Medical Education, 2007). This task is not done by some hospitals and even if the available beds are reported, there are still barriers. First, the statistics on available hospital beds may be inaccurate and under-reporting occurs. Secondly, in case these statistics are accurate, you still cannot be certain to find the beds unoccupied because it is not real time information, and perhaps shortly after the announcement of the statistics on available beds, the bed capacity in a hospital is reached. The supervision and unannounced visit of CTGI about hospital's empty beds, the use of other mechanisms for declaring available beds, such as a Bed manager, and use of new information technologies for the timely transmission of information can be proposed solutions to solve the barrier of accurate statistics for available beds (Abraham \& Reddy, 2010).

Web-based referral systems for inter-hospital transfers are effective mechanisms that show bed availability in 
hospitals. A web based system to identify optimal destination for patient transfer is proposed in university of Northern British Columbia which finds optimal hospital based on the selected criteria such as bed availability (Haque, Derksen, Calado, \& Foster, 2015).

\subsection{Monitoring}

Due to the different work processes in hospitals, especially non-educational hospitals, the admission process lasts longer than usual. Therefore, superior authorities must determine an identical process for non-educational hospitals and requirements must be considered for its implementation. In the past, an on-call physician in CTGI had strong legal authority to influence patient admission, but this has been eliminated today. Receiving hospital resists on patient admission even if it is ordered by CTGI and demand pretransfer coordination. This performance of CTGI indicates that it prefers scientific indications rather than bed indications. That is, if it had scientific indication but did not have an available bed, it would order patient transfer. Although CTGI is responsible for admission of the patients needing transfer based on the patient transfer protocols, it should not be done without coordination with the receiving hospital. Re-appointing of on call Physicians or delegating legal authority to the CTGI's Physicians and notifying the hospitals can be a strategy to overcome hospitals' resistance in case the patient transfer is done by CTGI.

Based on the findings of this study, it seems that not monitoring the performance of executive organizations, including private hospitals, in the area of patient transfer has caused fragmentation in patient care management. In the past, monitoring was done by the CTGI. In this approach, CTGI staff goes to hospitals randomly to examine the patient transfer standards using a patient transfer check list. Considering the ignorance of private hospital about patient transfer rules and the non-delivery of patient transfer forms, it seems that the previous authority of CTGI should be restored. Monitoring hospital performance through periodic reports from receiving hospitals can lead to better management. Strategies for overcoming weaknesses in enforcement of patient transfer rules include improving the monitoring system and accreditation concerning the process of patient transfer management.

\section{4 Feedback}

Giving feedback at several stages of the patient transfer process is important. These stages include sending reports from CTGI about its performance to superior levels, from CTGI to hospitals and from receiving hospitals to referring hospitals. There was no feedback in this study. Scientific evidence indicates that the lack of feedback about the mistakes happens in the patient transfer process and its implications can be inhibitors to change and barriers to improvement and promotion of services (Bitterman, 2000). If the receiving hospital gives feedback on the incorrect procedures to the referring hospital, it will negatively influence the management of subsequent transfers. In other words, it prevents the wrong actions that ultimately lead to secondary injuries to patients. Consistent with this study, a study conducted to evaluate the quality of patient transfer indicated the lack of a standard protocol for providing feedback to the referring physician as one of the causes of undesirable events occurring with transfer of patients (Ligtenberg et al., 2005). With efficient and effective legal support and also support from deputy treatment affair, it is possible to make the hospitals adopt transfer standards, such as scientific and regular feedback, and thus overcome the barriers of a lack of both coordination and feedback. One of the strategies for establishing the feedback system in CTGI can be setting up the audit system and designing standard patient transfer forms to evaluate the quantity and quality of the transfers and sending feedback to groups involved in patient transfer (Farley-Hills \& O'keeffe, 2010; Richardson, Malhotra, \& Kaushal, 2014). Meanwhile, it is possible to receive complaints of legal entities by adopting the appropriate methods in CTGI. The methods include a manual or electronic record system of complaints in order to achieve the final result and feedback. Informing the superior levels from wrong doings of the relevant units to and in return, the investigation of superior levels can also improve performances.

\subsection{Patient Transfer Consequences}

Despite the fact that patient transfer is done to provide better quality care, it may also be associated with undesirable events. The studies, which used a systematic review showed that consistent evidence on the perceived impact of communication breakdown on patient safety during intrahospital transfers (Hains, Marks, Georgiou, \& Westbrook, 2011; Ong \& Coiera, 2011).

Although there are potential risks and undesirable implications in patient transfer, the necessary measures must be taken to reduce these implications so that the patient can benefit from the advantages of transfer. These measures could include improving communication infrastructures and informing health care providers about the consequences of incorrect information and communication. A study in the Netherlands showed that $70 \%$ of secondary events and injuries occurred during transfer was preventable through better preparation and 
communication between hospital (Ligtenberg et al., 2005).

This study also had limitations. It was originally supposed to be a qualitative study using the FGD. However, the method of the study changed to individual interviews after three FGD meetings due to the poor presence of the invited people in the meetings caused by their working schedule. Perhaps, the involvement of practitioners in patient transfer from all levels including the CTGI and the referring and receiving hospitals together would lead to greater transparency in the expression of difficulties. Another limitation of the study was to choose only one referring hospital for interviews. If we could have selected several referring hospitals, some other barriers may have been expressed.

\section{Conclusion}

There are numerous barriers and shortcomings in communication and information exchange of patient transfer in CTGI and the referring and receiving hospitals due to the involvement of different units and individuals in the patient transfer. Poor communication and information exchange and inadequate monitoring lead to undesirable results and is followed by some threats to the health care system and patients. There is a need for proper monitoring by accountable organizations, a review of the protocols for patient transfer, to make people involved in patient transfer follow the protocols properly, an increase in intersectional collaboration, improvement in communications infrastructure and collection of information. The use of new information technologies can facilitate timely access to information on hospital beds' availability and clinical data of patients requiring transfer and prevent the undesirable events caused by poor communication and information exchange. To understand the objective and correct weaknesses and shortcomings of communication and information exchange in the patient transfer process, future studies need to be done using a qualitative method with approach for observational studies.

\section{Acknowledgements}

The authors would like to thank all participants of the study and the staff of Hospitals and Center for Treatment Guidance and Information for their collaboration.

\section{Competing Interests Statement}

The authors declare that there is no conflict of interests regarding the publication of this paper.

\section{References}

Abraham, J., \& Reddy, M. C. (2010). Challenges to inter-departmental coordination of patient transfers: A workflow perspective. Int J Med Inform, 79(2), 112-122. http://dx.doi.org/10.1016/j.ijmedinf.2009.11.001

Apker, J., Mallak, L. A., \& Gibson, S. C. (2007). Communicating in the "gray zone": Perceptions about emergency physician hospitalist handoffs and patient safety. Acad Emerg Med, 14(10), 884-894. http://dx.doi.org/10.1197/j.aem.2007.06.037

Behara, R., Wears, R. L., Perry, S. J., Eisenberg, E., Murphy, L., Vanderhoef, M., ... Cosby, K. (2005). A Conceptual Framework for Studying the Safety of Transitions in Emergency Care Advances in Patient Safety: From Research to Implementation (Volume 2: Concepts and Methodology). Rockville MD. PMID: 21249845 [PubMed].

Bitterman, R A. (2000). Transferring accepting patients under EMTALA. In: Bitterman RA ed. Providing Emergency Care under Federal Law: EMTALA Dallas Texas. American College of Emergency Physicians, 100-123.

Bosk, E. A., Veinot, T., \& Iwashyna, T. J. (2011). Which patients and where: A qualitative study of patient transfers from community hospitals. Med Care, 49(6), 592-598. http://dx.doi.org/10.1097/MLR.0b013e318 $20 \mathrm{fb} 71 \mathrm{~b}$

Coleman, E. A. (2003). Falling Through the Cracks: Challenges and Opportunities for Improving Transitional Care for Persons with Continuous Complex Care Needs. Journal of the American Geriatrics Society, 51(4), 549-555. http://dx.doi.org/10.1046/j.1532-5415.2003.51185.x

Coleman, E. A., Smith, J. D., Raha, D., \& Min, S. J. (2005). Posthospital medication discrepancies: Prevalence and contributing factors. Arch Intern Med, 165(16), 1842-1847. http://dx.doi.org/10.1001/archinte.165. 16.1842

Cwinn, M. A., Forster, A. J., Cwinn, A. A., Hebert, G., Calder, L., \& Stiell, I. G. (2009). Prevalence of information gaps for seniors transferred from nursing homes to the emergency department. Cjem, 11(5), 462-471. PMID: 19788791 [PubMed]. 
Dunnion, M. E., \& Kelly, B. (2008). All referrals completed? The issues of liaison and documentation when discharging older people from an emergency department to home. Journal of Clinical Nursing, 17(18), 2471-2479. PMID: 18988319 [PubMed]. http://dx.doi.org/10.1111/j.1365-2702.2007.02084.x

Farley-Hills, E., \& O'keeffe, S. (2010). Transfer of the critically ill adult: Building an infrastructure for Wales.

Gillman, L., Jacobs, I., \& Fatovich, D. M. (2014). Challenges in arranging interhospital transfer from a non-tertiary hospital emergency department in the Perth metropolitan area. Emerg Med Australas. http://dx.doi.org/10.1111/1742-6723.12299

Glintborg, B., Andersen, S. E., \& Dalhoff, K. (2007). Insufficient communication about medication use at the interface between hospital and primary care. Quality and Safety in Health Care, 16(1), 34-39. PMID: 17301202 [PubMed]. http://dx.doi.org/10.1136/qshc.2006.019828

Goldhill, D., Gemmel, L., Lutman, D., McDevitt, S., Parris, M., Waldmann, C., \& Dodds, C. (2009). Aagbi Safety Guideline: Interhospital Transfer. http://www.aagbi.org/sites/default//files/interhospital09.pdf

Griffiths, D., Morphet, J., Innes, K., Crawford, K., \& Williams, A. (2014). Communication between residential aged care facilities and the emergency department: A review of the literature. International Journal of Nursing Studies, 51(11), 1517-1523. http://dx.doi.org/10.1016/j.ijnurstu.2014.06.002

Haggstrom, M., Asplund, K., \& Kristiansen, L. (2009). Struggle with a gap between intensive care units and general wards. Int J Qual Stud Health Well-being, 4, 181-192. http://dx.doi.org/10.1080/17482620903 072508

Hains, I. M., Marks, A., Georgiou, A., \& Westbrook, J. I. (2011). Non-emergency patient transport: What are the quality and safety issues? A systematic review. International Journal for Quality in Health Care, 23(1), 68-75. http://dx.doi.org/10.1093/intqhe/mzq076

Halasyamani, L., Kripalani, S., Coleman, E., Schnipper, J., van Walraven, C., Nagamine, J., ... Manning, D. (2006). Transition of care for hospitalized elderly patients--development of a discharge checklist for hospitalists. J Hosp Med, 1(6), 354-360. http://dx.doi.org/10.1002/jhm.129

Haque, W., Derksen, B. A., Calado, D., \& Foster, L. (2015). Using business intelligence for efficient inter-facility patient transfer. Stud Health Technol Inform, 208, 170-176.

Horwitz, L. I., Meredith, T., Schuur, J. D., Shah, N. R., Kulkarni, R. G., \& Jenq, G. Y. (2009). Dropping the baton: A qualitative analysis of failures during the transition from emergency department to inpatient care. Ann Emerg Med, 53(6), 701-710.e704. http://dx.doi.org/10.1016/j.annemergmed.2008.05.007

Hustey, F. M., \& Palmer, R. M. (2010). An internet-based communication network for information transfer during patient transitions from skilled nursing facility to the emergency department. $J$ Am Geriatr Soc, 58(6), 1148-1152. http://dx.doi.org/10.1111/j.1532-5415.2010.02864.x

Hustey, F. M., \& Palmer, R. M. (2012). Implementing an Internet-based communication network for use during skilled nursing facility to emergency department care transitions: Challenges and opportunities for improvement. J Am Med Dir Assoc, 13(3), 249-253. http://dx.doi.org/10.1016/j.jamda.2010.07.003

Jeffs, L., Lyons, R. F., Merkley, J., \& Bell, C. M. (2013). Clinicians' views on improving inter-organizational care transitions. BMC Health Serv Res, 13, 289. http://dx.doi.org/10.1186/1472-6963-13-289

King, B. J., Gilmore-Bykovskyi, A. L., Roiland, R. A., Polnaszek, B. E., Bowers, B. J., \& Kind, A. J. (2013). The consequences of poor communication during transitions from hospital to skilled nursing facility: A qualitative study. J Am Geriatr Soc, 61(7), 1095-1102. http://dx.doi.org/10.1111/jgs.12328

Kripalani, S., LeFevre, F., Phillips, C. O., Williams, M. V., Basaviah, P., \& Baker, D. W. (2007). Deficits in communication and information transfer between hospital-based and primary care physicians: Implications for patient safety and continuity of care. Jama, 297(8), 831-841. PMID: 17327525 [PubMed]. http://dx.doi.org/10.1001/jama.297.8.831

Ligtenberg, J. J., Arnold, L. G., Stienstra, Y., van der Werf, T. S., Meertens, J. H., Tulleken, J. E., \& Zijlstra, J. G. (2005). Quality of interhospital transport of critically ill patients: A prospective audit. Crit Care, 9(4), R446-451. http://dx.doi.org/10.1186/cc3749

Mesteig, M., Helbostad, J. L., Sletvold, O., Rosstad, T., \& Saltvedt, I. (2010). Unwanted incidents during transition of geriatric patients from hospital to home: A prospective observational study. BMC Health Serv Res, 10, 1. http://dx.doi.org/10.1186/1472-6963-10-1 
Ministry of Health \& Medical Education. (2007). Republic Islamic of Iran.

Odetola, F. O., Bruski, L., Zayas-Caban, G., \& Lavieri, M. (2016). An Innovative Framework to Improve Efficiency of Interhospital Transfer of Children in Respiratory Failure. Ann Am Thorac Soc. http://dx.doi.org/10.1513/AnnalsATS.201507-401OC

Olsen, R. M., Ostnor, B. H., Enmarker, I., \& Hellzen, O. (2013). Barriers to information exchange during older patients' transfer: Nurses' experiences. J Clin Nurs, 22(19-20), 2964-2973. http://dx.doi.org/10.1111/jocn. 12246

Ong, M. S., \& Coiera, E. (2011). A systematic review of failures in handoff communication during intrahospital transfers. Jt Comm J Qual Patient Saf, 37(6), 274-284. PMID: 21706987 [PubMed].

Patterson, E. S., Roth, E. M., Woods, D. D., Chow, R., \& Gomes, J. O. (2004). Handoff strategies in settings with high consequences for failure: Lessons for health care operations. Int J Qual Health Care, 16(2), 125-132. http://dx.doi.org/10.1093/intqhc/mzh026

Payne, S., Kerr, C., Hawker, S., Hardey, M., \& Powell, J. (2002). The communication of information about older people between health and social care practitioners. Age and ageing, 31(2), 107-117. PMID: 12033184 [PubMed]. http://dx.doi.org/10.1093/ageing/31.2.107

Platts-Mills, T. F., Biese, K., LaMantia, M., Zamora, Z., Patel, L. N., McCall, B., ... Kizer, J. S. (2012). Nursing home revenue source and information availability during the emergency department evaluation of nursing home residents. J Am Med Dir Assoc, 13(4), 332-336. http://dx.doi.org/10.1016/j.jamda.2010.12.009

Richardson, J. E., Malhotra, S., \& Kaushal, R. (2014). A case report in health information exchange for inter-organizational patient transfers. Appl Clin Inform, 5(3), 642-650. http://dx.doi.org/10.4338/ACI-2014 -02-CR-0016

Shah, F., Burack, O., \& Boockvar, K. S. (2010). Perceived barriers to communication between hospital and nursing home at time of patient transfer. J Am Med Dir Assoc, 11(4), 239-245. http://dx.doi.org/10.1016/ j.jamda.2009.08.006

Shanley, C., Sutherland, S., Stott, K., Tumeth, R., \& Whitmore, E. (2008). Increasing the profile of the care of the older person in the ED: A contemporary nursing challenge. International Emergency Nursing, 16(3), 152-158. http://dx.doi.org/10.1016/j.ienj.2008.05.005

Terrell, K. M., \& Miller, D. K. (2006). Challenges in transitional care between nursing homes and emergency departments. J Am Med Dir Assoc, 7(8), 499-505. http://dx.doi.org/10.1016/j.jamda.2006.03.004

Terrell, K. M., Brizendine, E. J., Bean, W. F., Giles, B. K., Davidson, J. R., Evers, S., ... Cordell, W. H. (2005). An extended care facility-to-emergency department transfer form improves communication. Acad Emerg Med, 12(2), 114-118. http://dx.doi.org/10.1197/j.aem.2004.10.013

Thraen, I., Bair, B., Mullin, S., \& Weir, C. R. (2012). Characterizing "information transfer" by using a Joint Cognitive Systems model to improve continuity of care in the aged. Int J Med Inform, 81(7), 435-441. http://dx.doi.org/10.1016/j.jimedinf.2011.11.006

Warren, J., Fromm, R. E. Jr., Orr, R. A., Rotello, L. C., \& Horst, H. M. (2004). Guidelines for the inter-and intrahospital transport of critically ill patients. Crit Care Med, 32(1), 256-262. http://dx.doi.org/10.1097/01. CCM.0000104917.39204.0A

\section{Copyrights}

Copyright for this article is retained by the author(s), with first publication rights granted to the journal.

This is an open-access article distributed under the terms and conditions of the Creative Commons Attribution license (http://creativecommons.org/licenses/by/3.0/). 